\title{
Gesamtliteraturverzeichnis
}

Adler/Düring/Schmaltz, Rechnungslegung und Prüfung der Unternehmen, 6. Aufl. 1994 ff.

Apitz/Bruschke, Der GmbH-Jahresabschluss, Loseblatt (Stand Mai 2020)

Baetge/Kirsch/Thiele, Bilanzen, 15. Aufl. 2019

Baetge/Kirsch/Thiele (Hrsg.), Bilanzrecht, Loseblatt (Stand Mai 2020)

Baetge/Kirsch/Thiele, Konzernbilanzen, 13. Aufl. 2019

Ballwieser/Coenenberg/v. Wysocki (Hrsg.), Handwörterbuch der Rechnungslegung und Prüfung, 3. Aufl. 2002

Baumbach/Hopt, HGB, 39. Aufl. 2020

Baumbach/Hueck, GmbHG, 18. Aufl. 2006, 22. Aufl. 2019

Beck'scher Bilanzkommentar, hrsg. v. Grottel/Schmidt/Schubert/Störk, 12. Aufl. 2020

Beck'sches Handbuch der Personengesellschaften, hrsg. v. Prinz/Kahle, 5. Aufl. 2020

Beck'sches Handbuch der Rechnungslegung, hrsg. v. Böcking/Gros/Oser/Scheffler/Thormann, Loseblatt

(Stand März 2020)

Beck'sches IFRS-Handbuch, Kommentierung der IFRS/IAS, hrsg. v. Brune/Driesch/Schulz-Danso/Senger,

6. Aufl. 2020

Beck'scher Online-Kommentar HGB, hrsg. v. Häublein/Hoffmann-Theinert

beck-online Großkommentar, hrsg. v. Henssler

Bertram/Brinkmann/Kessler/Müller, Haufe HGB Bilanz-Kommentar, 10. Aufl. 2019

Bieg/Kußmaul/Waschbusch, Externes Rechnungswesen, 6. Aufl. 2012

Bitz/Schneeloch/Wittstock/Patek, Der Jahresabschluss, 6. Aufl. 2014

Blümich, EStG - KStG - GewStG mit Nebengesetzen, Loseblatt (Stand März 2020)

Bolin/Dreyer/Schäfer (Hrsg.), Handbuch Handelsrechtliche Rechnungslegung, 2013

Bordewin/Brandt, Einkommensteuergesetz, Loseblatt (Stand März 2020)

Bork/Schäfer, GmbHG, 4. Aufl. 2019

Brönner/Bareis/Hahn/Maurer/Poll/Schramm (Hrsg.), Die Bilanz nach Handels- und Steuerrecht, 11. Aufl. 2016

Budde/Moxter/Offerhaus (Hrsg.), Handelsbilanzen und Steuerbilanzen - Festschrift Beisse, 1997

Bürgers/Körber, Aktiengesetz, 4. Aufl. 2017

Busse von Colbe/Ordelheide/Gebhard/Pellens, Konzernabschlüsse, 9. Aufl. 2009

Coenenberg/Haller/Schultze, Jahresabschluss und Jahresabschlussanalyse, 25. Aufl. 2018

Claussen/Scherrer (Hrsg.), Kölner Kommentar zum Rechnungslegungsrecht, 2011

Dötsch/Pung/Möhlenbrock (Hrsg.), Die Körperschaftsteuer, Loseblatt (Stand Feb. 2020)

Ebenroth/Boujong/Joost/Strohn (Hrsg.), HGB, Bd. 1 ( $\$ \$ 1-342 \mathrm{e})$, 4. Aufl. 2020

Eilers/Rödding/Schmalenbach (Hrsg.), Unternehmensfinanzierung, 2. Aufl. 2014

Eisele/Knobloch, Technik des betrieblichen Rechnungswesens, 9. Aufl. 2019

Eisgruber, Umwandlungssteuergesetz, 2. Aufl. 2018

Emmerich/Habersack, Aktien- und GmbH-Konzernrecht, 9. Aufl. 2019

Emmerich/Habersack, Konzernrecht, 11. Aufl. 2020

Ensthaler (Hrsg.), HGB, 8. Aufl. 2015

Erle/Sauter (Hrsg.), Körperschaftsteuergesetz, 3. Aufl. 2010

Ernst \& Young (Hrsg.), Körperschaftsteuergesetz, Loseblatt

Farr/Niemann/Bruckner, Jahresabschlusserstellung, 3. Aufl. 2016

Federmann/Kußmaul/Müller (Hrsg.), Handbuch der Bilanzierung, Loseblatt

Frotscher/Drüen, KStG - GewStG - UmwStG, Loseblatt (Stand Apr. 2020)

Frotscher/Geurts, EStG, Loseblatt (Stand Apr. 2020)

Gehrlein/Born/Simon, GmbHG, 4. Aufl. 2019

Geßler, AktG, Loseblatt

Glade, Praxishandbuch der Rechnungslegung und Prüfung, 2. Aufl. 1995

Gosch (Hrsg.), Körperschaftsteuergesetz, 3. Aufl. 2015

Gräfer, Rechnungslegung, 5. Aufl. 2016

Grigoleit, AktG, 2013 
Großfeld/Luttermann, Bilanzrecht, 4. Aufl. 2005

Großkommentar zum AktG, hrsg. v. Hirte/Mülbert/Roth, Bd. 1, 5, 5. Aufl. 2016 ff.

Großkommentar zum HGB, begr. v. Staub, hrsg. von Canaris/Habersack/C. Schäfer, Bd. 5-7, 5. Aufl. 2010 ff.

Haag/Löffler (Hrsg.), HGB, 2. Aufl. 2014

Haase/Hruschka (Hrsg.), UmwStG, 2. Aufl. 2017

Haritz/Menner/Bilitewski (Hrsg.), Umwandlungssteuergesetz, 5. Aufl. 2019

Haun/Kahle/Goebel/Reiser (Hrsg.), Außensteuergesetzt, Kommentar, Loseblatt

Heidel/Schall (Hrsg.), HGB, 3. Aufl. 2019

Helmrich, Bilanzrichtlinien-Gesetz, 1986

Hennrichs, Wahlrechte im Bilanzrecht der Kapitalgesellschaften, 1999

Henssler/Strohn, Gesellschaftsrecht, 4. Aufl. 2019

Herrmann/Heuer/Raupach, EStG - KStG, hrsg. v. Hey/Klein/Wendt, Loseblatt (Stand März 2020)

Hesselmann/Tillmann/Mueller-Thuns (Hrsg.), Handbuch GmbH \& Co. KG, 22. Aufl. 2020

Heuser/Theile, IFRS-Handbuch, 6. Aufl. 2019

Hofbauer/Kupsch (Hrsg.), Rechnungslegung, Loseblatt (Stand Mai 2020)

Hoffmann/Lüdenbach, NWB Kommentar Bilanzierung, 11. Aufl. 2019

Hölters, AktG, 3. Aufl. 2017

Hopt/Merkt, Bilanzrecht, 2010

Heymann-Handelsgesetzbuch, hrsg. v. Horn/Balzer/Borges/Herrmann, Drittes Buch (\$\$238-342e),

3. Aufl. 2019

Horschitz/Groß/Fanck, Bilanzsteuerrecht und Buchführung, 15. Aufl. 2018

Hüffer/Koch, AktG, 14. Aufl. 2020

Icking, Die Rechtsnatur des Handelsbilanzrechts, 2000

IDW (Hrsg.), WP Handbuch, 16. Aufl. 2019

Jacobs/Scheffler/Spengel (Hrsg.), Unternehmensbesteuerung und Rechtsform, 5. Aufl. 2015

Jauernig, Bürgerliches Gesetzbuch (BGB), Kommentar, 17. Aufl. 2018

Kanzler/Kraft/Bäuml/Hechtner/Marx (Hrsg.), Einkommensteuergesetz, 5. Aufl. 2020

Kirchhof (Hrsg.), EStG, 19. Aufl. 2020

Kirchhof/Söhn/Mellinghoff (Hrsg.), Einkommensteuergesetz, Loseblatt (Stand Mai 2020)

Kirsch (Hrsg.), Rechnungslegung, Loseblatt (Stand Mai 2020)

Knobbe-Keuk, Bilanz- und Unternehmenssteuerrecht, 9. Aufl. 1993

Kölner Kommentar zum Aktiengesetz, hrsg. v. Zöllner/Noack, 3. Aufl. 2004 ff.

Kölner Kommentar zum Rechnungslegungsrecht, hrsg. v. Claussen/Scherrer, 2011

Koller/Kindler/Roth/Drüen, Handelsgesetzbuch: HGB, 9. Aufl. 2019

Korn (Hrsg.), Einkommensteuergesetz, Loseblatt

Kraft/Edelmann/Bron (Hrsg.), Umwandlungssteuergesetz, 2. Aufl. 2019

Kruse, Grundsätze ordnungsmäßiger Buchführung, 3. Aufl. 1970/1978

Kuhn/Hachmeister, Rechnungslegung und Prüfung von Finanzinstrumenten, 2015

Künkele/Zwirner (Hrsg.), Bilanzierung bei Personengesellschaften, 2. Aufl. 2016

Küting/Weber (Hrsg.), Handbuch der Rechnungslegung - Einzelabschluss, Loseblatt (Stand März 2020)

Küting/Weber, Die Bilanzanalyse, 11. Aufl. 2015

Küting/Weber, Der Konzernabschluss, 14. Aufl. 2018

Küting/Weber, Handbuch der Konzernrechnungslegung, 2. Aufl. 1998

Lademann, EStG, Loseblatt (Stand Mai 2020)

Lademann, KStG, Loseblatt (Stand Nov. 2019)

Leffson, Die Grundsätze ordnungsmäßiger Buchführung, 7. Aufl. 1987

Leffson/Rückle/Großfeld (Hrsg.), Handwörterbuch unbestimmter Rechtsbegriffe im Bilanzrecht des HGB, 1986 Littmann/Bitz/Pust (Hrsg.), Das Einkommensteuerrecht, Loseblatt (Stand Apr. 2020)

Lutter/Hommelhoff (Hrsg.), GmbH-Gesetz, 20. Aufl. 2020

Merkt, Unternehmenspublizität, 2001

Merkt/Probst/Fink, Rechnungslegung nach HGB und IFRS, 2017

Michalski/Heidinger/Leible/Schmidt, GmbH-Gesetz, 3. Aufl. 2017

Mössner/Seeger/Oellerich (Hrsg.), Körperschaftsteuergesetz, 4. Aufl. 2019 
Moxter, Bilanzlehre, Bd. I und Bd. II, 3. Aufl. 2012

Moxter, Bilanzrechtsprechung, 6. Aufl. 2007

Moxter, Grundsätze ordnungsgemäßer Rechnungslegung, 2003

Münchener Handbuch des Gesellschaftsrechts, Bd. 3: Gesellschaft mit beschränkter Haftung, hrsg. v. Priester/D. Mayer/Wicke, 5. Aufl. 2018; Bd. 4: Aktiengesellschaft, hrsg. v. Hoffmann-Becking, 4. Aufl. 2015; Bd. 8: Umwandlungsrecht, hrsg. v. Lieder/Wilk/Ghassemi-Tabar, 5. Aufl. 2018

Münchener Kommentar zum Aktiengesetz, hrsg. v. Goette/Habersack/Kalss, 3. Aufl. 2008 ff., 4. Aufl. 2014 ff., 5. Aufl. $2019 \mathrm{ff}$.

Münchener Kommentar zum Bilanzrecht, hrsg. v. Hennrichs/Kleindiek/Watrin, Bd. 2 (\$\$ 238-342e), 2013

Münchener Kommentar zum GmbHG, hrsg. v. Fleischer/Goette, 3. Aufl. 2018 ff.

Münchener Kommentar zum HGB, hrsg. v. Karsten Schmidt, 3. Aufl. 2013 ff., 4. Aufl. 2016 ff.

Oetker (Hrsg.), HGB, 6. Aufl. 2019

Palandt, BGB, 79. Aufl. 2020

Pelka/Petersen (Hrsg.), Beck'sches Steuerberater-Handbuch 2019/2020, 17. Aufl. 2019

Petersen/Zwirner, Handbuch Bilanzrecht, 2. Aufl. 2018

Petersen/Zwirner/Brösel (Hrsg.), Systematischer Praxiskommentar Bilanzrecht, 4. Aufl. 2020

Prinz/Kanzler (Hrsg.), Handbuch Bilanzsteuerrecht, 3. Aufl. 2018

Rödder/Herlinghaus/Neumann (Hrsg.), KStG, 2015

Rödder/Herlinghaus/van Lishaut (Hrsg.), UmwStG, 3. Aufl. 2019

Roth/Altmeppen, GmbHG, 9. Aufl. 2019

Rowedder/Schmidt-Leithoff, GmbHG, 6. Aufl. 2017

Russ/Janßen/Götze, BilRUG - Auswirkungen auf das deutsche Bilanzrecht, 2015

Saenger/Inhester, GmbHG, 4. Aufl. 2020

Schäfer (Hrsg.), Publizitätsgesetz, NOMOS - Das Deutsche Bundesrecht - Erläuterungen, 2. Aufl. 2016

Scheffler, Besteuerung von Unternehmen, Bd. 2, Steuerbilanz, 9. Aufl. 2018

Scherrer, Konzernrechnungslegung nach HGB, 3. Aufl. 2012

Schiffers/Theile, Bilanzrecht der GmbH, 2016

Schildbach/Feldhoff, Der Konzernabschluss nach HGB und IFRS, 8. Aufl. 2018

Schmidt, EStG, hrsg. v. Weber-Grellet, 39. Aufl. 2020

Schmidt, Karsten, Handelsrecht, 6. Aufl. 2014

Schmidt, Karsten/Lutter, AktG, 4. Aufl. 2020

Schmidt, M./Prinz, BilRUG in der Praxis, 2016

Schmitt/Hörtnagl/Stratz, UmwG - UmwStG, 8. Aufl. 2018

Schneider, Grundzüge der Unternehmensbesteuerung, 6. Aufl. 1994

Schnitger/Fehrenbacher (Hrsg.), Kommentar Körperschaftsteuer, 2. Aufl. 2018

Scholz, GmbHG, 11. Aufl. 2012 ff., 12. Aufl. 2018 ff.

Schön, Steuerliche Maßgeblichkeit in Deutschland und Europa, 2005

Schreiber, Besteuerung der Unternehmen, 4. Aufl. 2017

Schulze-Osterloh/Hennrichs/Wüstemann/Wüstemann (Hrsg.), Handbuch des Jahresabschlusses, Loseblatt (Stand März 2020)

Spindler/Stilz (Hrsg.), AktG, 4. Aufl. 2019

Streck (Hrsg.), KStG, 9. Aufl. 2018

Tipke/Lang, Steuerrecht, 23. Aufl. 2018

Ulmer, HGB-Bilanzrecht, 2002 (Reprint 2015)

Ulmer/Habersack/Löbbe, GmbHG Großkommentar (Bd. II \$\$ 29-52), 2. Aufl. 2014

Weber-Grellet, Bilanzsteuerrecht, 18. Aufl. 2020

Westermann/Wertenbruch, Handbuch Personengesellschaften, Loseblatt (Stand März 2020)

Wicke, GmbHG, 3. Aufl. 2016

Widmann/Mayer (Hrsg.), Umwandlungsrecht, Loseblatt

Wiedmann/Böcking/Gros, Bilanzrecht, 4. Aufl. 2019

Winkeljohann/Förschle/Deubert (Hrsg.), Sonderbilanzen, 5. Aufl. 2016

Winkeljohann/Schäfer, Latente Steuern, 2015 
Winnefeld, Bilanz-Handbuch, 5. Aufl. 2015

Wöhe/Mock, Die Handels- und Steuerbilanz, 6. Aufl. 2010

Ziemons/Binnewies, Handbuch Aktiengesellschaft, Loseblatt (Stand März 2020)

\section{Abgekürzt zitierte Literatur}

ADS

Beck BilKomm.

Beck HdB PersG

Beck HdR

BeckOGK

BeckOK HGB

BHR

$B K T$

GK

Großkomm.

Großkomm. AktG

Haufe BilKomm.

HdJ

HdKR

HdR

HHR

HuRB

Kölner Komm. AktG Kölner Komm. RLR KSM

MünchHdb. GesR MünchKomm. AktG

MünchKomm. BilR

MünchKomm. GmbHG MünchKomm. HGB

Nomos-BR
Adler/Düring/Schmaltz, Rechnungslegung und Prüfung der Unternehmen, 6. Aufl. $1994 \mathrm{ff}$.

Grottel/Schmidt/Schubert/Störk (Hrsg.), Beck'scher Bilanzkommentar, 12. Aufl. 2020 Prinz/Kahle (Hrsg.), Beck'sches Handbuch der Personengesellschaften, 5. Aufl. 2020

Böcking/Gros/Oser/Scheffler/Thormann, Beck'sches Handbuch der Rechnungslegung, Loseblatt (Stand März 2020)

Henssler (Hrsg.), beck-online Großkommentar

Häublein/Hoffmann-Theinert (Hrsg.), Beck'scher Online-Kommentar HGB Hofbauer/Kupsch (Hrsg.), Rechnungslegung, Loseblatt (Stand Mai 2020) Baetge/Kirsch/Thiele

Ulmer/Habersack/Löbbe (Hrsg.), GmbHG Großkommentar (Bd. II \$\$ 29-52), 2. Aufl. 2014

Staub, Großkommentar zum HGB, hrsg. von Canaris/Habersack/C. Schäfer, 5. Aufl. $2010 \mathrm{ff}$.

Hirte/Mülbert/Roth (Hrsg.), Großkommentar zum AktG, 5. Aufl. 2016 ff.

Bertram/Brinkmann/Kessler/Müller, Haufe HGB Bilanz-Kommentar, 10. Aufl. 2019

Schulze-Osterloh/Hennrichs/Wüstemann/Wüstemann (Hrsg.), Handbuch des Jahresabschlusses, Loseblatt (Stand März 2020)

Küting/Weber, Handbuch der Konzernrechnungslegung, 1998

Küting/Weber (Hrsg.), Handbuch der Rechnungslegung - Einzelabschluss, Loseblatt (Stand März 2020)

Herrmann/Heuer/Raupach, EStG - KStG, Loseblatt (Stand März 2020)

Leffson/Rückle/Großfeld (Hrsg.), Handwörterbuch unbestimmter Rechtsbegriffe im Bilanzrecht des HGB, 1986

Zöllner/Noack (Hrsg.), Kölner Kommentar zum AktG, 3. Aufl. 2004 ff. Claussen/Scherrer (Hrsg.), Kölner Kommentar zum Rechnungslegungsrecht, 2011 Kirchhof/Söhn/Mellinghoff(Hrsg.), Einkommensteuergesetz, Loseblatt (Stand Mai 2020)

Münchener Handbuch des Gesellschaftsrechts, 4. Aufl. 2015 ff.

Münchener Kommentar zum Aktiengesetz, 3. Aufl. 2008 ff., 4. Aufl. 2014 ff., 5. Aufl. 2019 ff.

Hennrichs/Kleindiek/Watrin (Hrsg.), Münchener Kommentar zum Bilanzrecht, Bd. 2 (\$\$ 238-342e), 2013

Münchener Kommentar zum GmbHG, 3. Aufl. 2018 ff.

Münchener Kommentar zum HGB, 3. Aufl. 2013 ff., 4. Aufl. 2016 ff.

Schäfer (Hrsg.), Publizitätsgesetz, NOMOS - Das Deutsche Bundesrecht Erläuterungen, 2. Aufl. 2016 